\title{
Spectroelectrochemical Studies on the Oxidation Pathway of Epinephrine
}

\author{
Suk Han KIM and In-Hyeong Yeo
}

Department of Chemistry, Dongguk University, Seoul 100-715, Korea

\begin{abstract}
Oxidation of epinephrine at a gold electrode was studied in acidic solutions by a near normal incidence reflectance spectroelectrochemical (NNIRS) system. Also, cyclic voltammetric(CV) data were used to interpret the oxidation pathway of epinephrine. Some spectroscopic results are in good agreement with the previous reports at a carbon paste electrode. However, assignment of each peak in the CVs is not always in good agreement with the past findings, especially the formation of dehydrated form of leucoadrenochrome is not likely to occur under acidic conditions
\end{abstract}

keywords Spectroelectrochemical, epinephrine, adrenochrome, adrenalinequinone, leucoadrenochrome

Electrochemical characteristics of catecholamine and its derivatives have been studied by many groups. $^{1-5}$ Epinephrine, one of hormones of the adrenal medullar, was found to have profound effects on the body metabolism. For many years, it is known that various effects caused by stimulation of sympathetic nerves were due to the release of epinephrine and its derivatives from the nerve.

The oxidation pathway of epinephrine is known to be involved in enzymatic and non-enzymatic processes. Due to easy oxidation of epinephrine under the physiological $\mathrm{pH}$ condition, the oxidation of epinephrine has not been clearly revealed and remains in a controversial matter. The first mechanistic study on the electrochemical oxidation of epinephrine was done at a planar carbon paste electrode by cyclic voltammetry and chronoamperometry. ${ }^{6}$ At carbon electrodes, oxidation of epinephrine proceeds through two electron process and the rate of one electron transfer is slower than that observed at metal electrodes. It was suggested that the over-all oxidation of epinephrine followed an ECC(electron transfer- chemical reaction- chemical reaction) mechanism, which was different from the ECE model in their previous paper ${ }^{7}$ although the two models give the same results qualitatively.

In this communication, the oxidation of epinephrine was studied by a NNIRS system and electrochemical techniques on a gold electrode in acidic solutions. Light absorbing species generated at the gold electrode by applying a potential reveal some new findings. Some of our results are in part consistent with the previous findings. The same and differences between this study and the previous ones will be discussed based on in situ spectroscopic data recorded with electrochemical measurements.

\section{Experimental}

\section{Reagents}

Epinephrine obtained from Sigma Chemical Co. was used as received. Aqueous solutions were prepared by using purified water by a Milli-Q purification system. All inorganic salts for electrolytes were of reagent grade.

\section{Apparatus}

The spectroelectrochemical system, a near normal incidence reflectance mode, which is very similar to that of previously reported one ${ }^{8}$ was assembled in this laboratory. The schematic diagram of the experimental setup is shown in Fig. 1. The electrochemical components were just like an ordinary three electrode system. A high reflective gold electrode(Pine disk electrode) was used as a working electrode placing the optical fiber unit above it. The reference electrode put into a Luggin capillary was $\mathrm{Ag} / \mathrm{AgCl}$ in a saturated $\mathrm{KCl}$ solution. All potentials are referred to this electrode. The counter electrode was a spiral type platinum wire. Electrochemical measurements were made under semi- infinite diffusion conditions or thin layer type by adjusting the distance between the working electrode and the optical probe unit. The whole part of the electrochemical cell was encapsulated into a light-tight box made of wood 
and painted a black inside to prevent scattered radiation from outside.

The spectroscopic system consists of $150 \mathrm{~W}$ Xe lamp, holographic monochromator of 1200 lines $/ \mathrm{mm}$, a bifurcated optical fiber, and PMT as a detector of 185$870 \mathrm{~nm}$ range. The bifurcated optical probe unit was immersed into a solution of transparent silicone sealer in a tube, whose one of the end was sealed with a quartz slide for the spectral measurements.

\section{Measurements}

Cyclicvoltammograms of $3.3 \mathrm{mM}$ epinephrine were obtained in $0.1 \mathrm{M} \mathrm{H}_{2} \mathrm{SO}_{4}$ and in ammonium acetate buffer solution of $\mathrm{pH} 3.0$ and 4.8 at the high reflective gold electrode. The CVs were recorded under various scan rates, different potential windows and various starting potentials.

There is a series of measurements to get a series of spectra under different experimental conditions. The absorption spectra were recorded while the applied potential to the working electrode was stepped from a double layer potential region to a positive potential enough to oxidize the epinephrine. Also, absorption spectra were recorded at a certain wavelength while the applied potential of the working electrode was scanning to get a species selective absorption spectrum. After exhaustive electrolysis in the spectroelectrochemical cell (the applied potential for the electrolysis was $700,650,550 \mathrm{mV}$ in each $\mathrm{pH}$ solution), a series of spectra was recorded with time at an open circuit potential or by applying carefully selected potentials. Therefore, the difference spectra could be obtained by subtracting from absorption bands at an applied potential to absorption bands at an open potential.

\section{Results and Discussion}

The interesting features of the CVs of epinephrine are in that the number and shapes of peaks are very much dependent on $\mathrm{pH}$ and working electrode materials. A CV of $3.3 \mathrm{mM}$ epinephrine in $0.1 \mathrm{M}$ $\mathrm{H}_{2} \mathrm{SO}_{4}$ solution at an Au electrode is shown in Fig. 2. The first oxidation peak at $570 \mathrm{mV}$ in the forward scan and the corresponding reduction peak at $510 \mathrm{mV}$ in the reverse scan were observed. The two peaks correspond to the oxidation and reduction of quinone and hydroquinone moiety of the epinephrine. This kind of behaviors was also observed at a planar carbon paste electrode by Hawley et. al. ${ }^{6}$

They presented a mechanism for the oxidation of epinephrine, which is schematically represented as follows.

$$
\begin{aligned}
& \mathrm{AD} \rightarrow \mathrm{ADQH}+2 \mathrm{H}^{+}+2 \mathrm{e} \\
& \mathrm{ADQH} \rightarrow \mathrm{ADQ}+\mathrm{H}^{+} \\
& \mathrm{ADQ} \rightarrow \mathrm{LAD} \\
& \mathrm{LAD}+\mathrm{ADQ} \rightarrow \mathrm{AD}+\mathrm{ADQC} \\
& \mathrm{LAD} \rightarrow \mathrm{IND}+\mathrm{H}_{2} \mathrm{O}
\end{aligned}
$$

AD represents epinephrine whose name is also called adrenaline. $A D Q H$ and $A D Q$ are the first oxidation product with $\mathrm{N}$-protonated and deprotonated forms of adrenalinequinone, respectively. LAD and ADQC correspond to leucoadrenochrome and adrenochrome, respectively. IND represents the dehydrated form of LAD. As shown in the reactions above $A D$ is oxidized to $\mathrm{ADQH}$ or $\mathrm{ADQ}$ depending on the $\mathrm{pH}$ of the solution. ADQ proceed a chemical reaction to form LAD, which is easily oxidized by ADQ and LAD can convert IND by loosing water molecule.

In ammonium acetate buffer solution of $\mathrm{pH} 3.0$, two cathodic peaks $(\mathrm{C1}, \mathrm{C} 2)$ and three anodic peaks(A1, A2, A3) as shown in Fig. 3a were observed. In pH 4.8 solution one cathodic peak $(\mathrm{Cl})$ and three anodic peaks(A1, A2, A3) as shown in Fig. 3b were

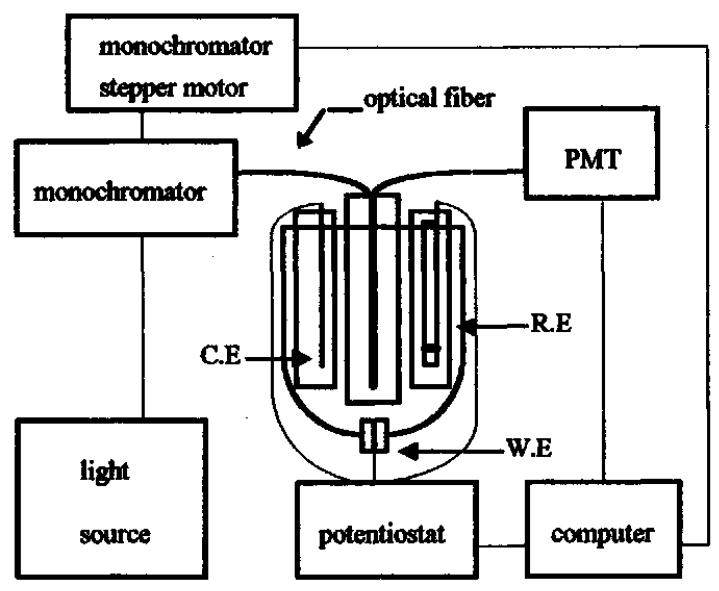

Fig. 1 Schematic diagram of experimental setup

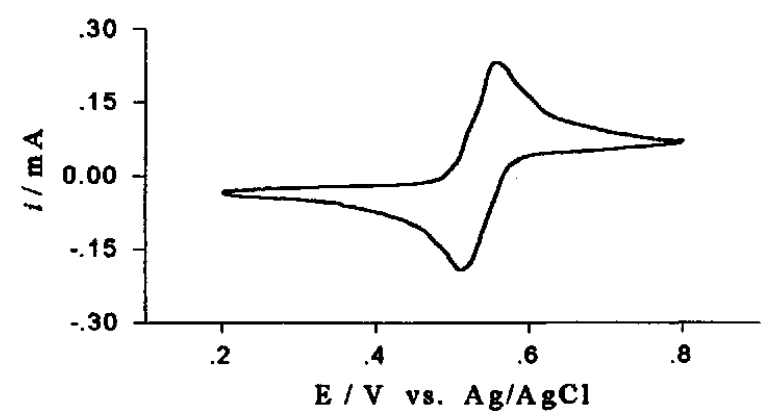

Fig. 2 Cyclicvoltammogram of $3.3 \mathrm{mM}$ epinephrine at an Au electrode in $0.1 \mathrm{M} \mathrm{H}_{2} \mathrm{SO}_{4}$. Scan rate; $50 \mathrm{mV} / \mathrm{sec}$ 
observed. The A3 peak is responsible for the oxidation of epinephrine and $\mathrm{C} 2$ corresponds to the reduction of the oxidation products on the subsequent scan. In pH 4.8 solution it was observed that the cathodic peak corresponding to $\mathrm{C} 2$ in $\mathrm{pH} 3.0$ solution disappeared and only one cathodic peak, $\mathrm{Cl}$, at a more negative potential appeared. This suggests that the first oxidation product undergoes chemical reaction quickly in higher $\mathrm{pH}$ solution and the product obtained following chemical step is more stable than the first oxidation product of epinephrine, ADQ or $\mathrm{ADQH}$. The half-life of ADQ was estimated to be $0.7 \mathrm{~s}$ at a solution of $\mathrm{pH} 5 .^{9}$ If potential scan starts from the potential below an onset potential of $A 3$, then, the cathodic peak $(\mathrm{Cl})$ and the anodic peaks, $\mathrm{Al}$ and $\mathrm{A} 2$, are not observed in the first scan. Once the applied potential is higher than that of first oxidation of epinephrine, then, the $\mathrm{Cl}$ is generated and the corresponding two anodic peaks appears in the second and more scans. This strongly implies that the $\mathrm{Al}$ and A2 waves are due to reoxidation of the reduction products responsible for $\mathrm{Cl}$. It should be noted that the relative height of peaks $\mathrm{C} 1$ and $\mathrm{C} 2$ changes with scan rates. The peak, $\mathrm{C} 2$ is greater than $\mathrm{Cl}$ at a faster scan rate indicating that less time is given for occurring chemical reactions and the smaller $\mathrm{Cl}$ peak results.

A series of spectra was recorded when a positive potential or no potential was applied to monitor any light absorbing species as intermediates or products. When the epinephrine in $0.1 \mathrm{M} \mathrm{H}_{2} \mathrm{SO}_{4}$ is oxidized at $700 \mathrm{mV}$ at a gold electrode, a recorded spectrum is shown in Fig. 4a which has two absorption bands at 290 and $380 \mathrm{~nm}$. Shown in Fig. 4b appearing one absorption band at $290 \mathrm{~nm}$ is the spectrum when no potential is applied, i.e., an open circuit potential, in the presence of epinephrine. Therefore, it is believed that the first oxidation product, $\mathrm{ADQH}$ shows an absorption band at $380 \mathrm{~nm}$ due to $\mathrm{n}-\pi^{*}$ transition of carbonyl group. The absorption maximum at $\mathbf{3 8 0}$ $\mathrm{nm}$ and smaller band at $525 \mathrm{~nm}$ indicates the formation of a new reaction product, ADQH.

If the applied potential is changed from $700 \mathrm{mV}$ to an open circuit the spectral feature returns from $4 a$ to $4 \mathrm{~b}$, indicating that the $\mathrm{ADQH}$ is unstable and goes quickly back to that of epinephrine through an rearrangement reaction in this condition. Therefore, it can be deduced that the heavily protonated quinones are hard to undergo cyclization reaction ${ }^{7}$ or be stabilized because of easy conversion to epinephrine.

When we ran the same experiments in $\mathrm{pH} 3.0$ and 4.8 ammonium acetate buffer solution, different absorption bands were observed as shown in Fig. 5 .
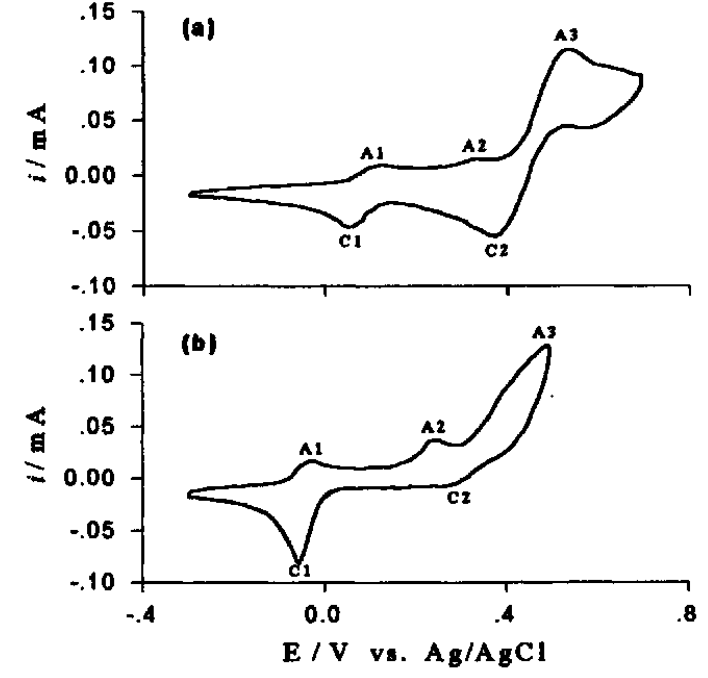

Fig. 3 Cyclicvoltammograms of $3.3 \mathrm{mM}$ epinephrine at an Au electrode (a) in pH 3.0 buffer solution (b) in $\mathbf{~ p H}$ buffer solution. Scan rate; $50 \mathrm{mV} / \mathrm{sec}$

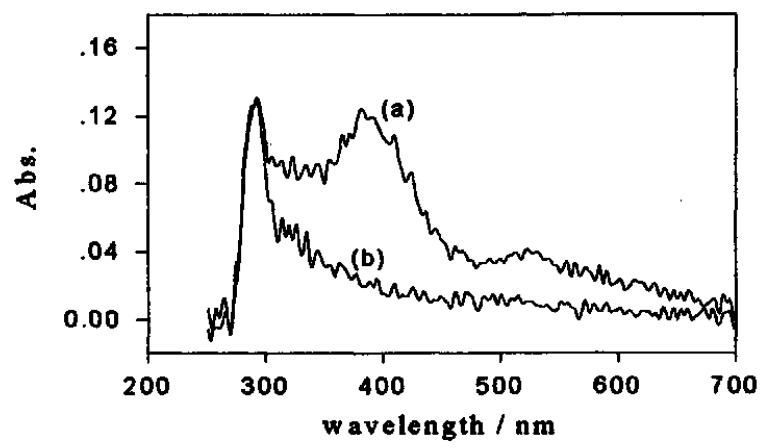

Fig. 4 Absorption spectra recorded by applying (a) 700 $\mathrm{mV}$, (b) no potential in $0.1 \mathrm{M} \mathrm{H}_{2} \mathrm{SO}_{4}$ containing $3.3 \mathrm{mM}$ epinephrine

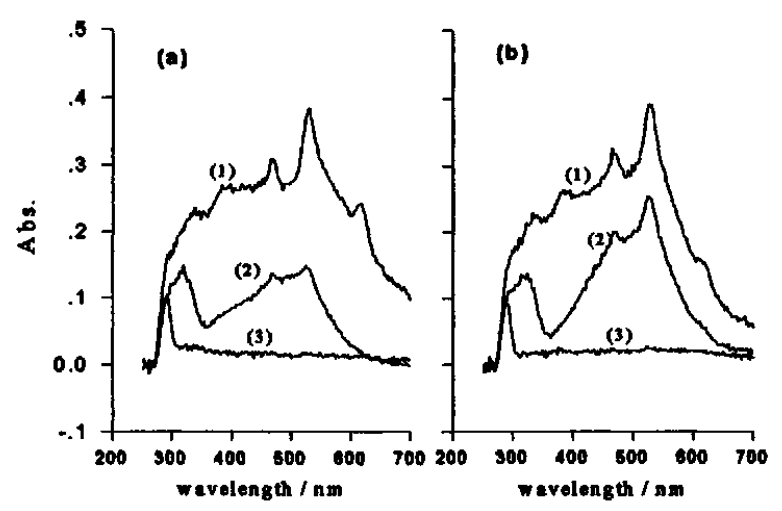

Fig. 5 Absorption spectra recorded by applying potentials in pH 3.0 buffer (1) $200 \mathrm{mV}$ after bulk electrolysis at $650 \mathrm{mV}$ (2) $650 \mathrm{mV}$ (3) no potential; (b) in pH 4.8 buffer, (1) $50 \mathrm{mV}$ after bulk electrolysis at $550 \mathrm{mV}$ (2) $550 \mathrm{mV}$ (3) no potential. 


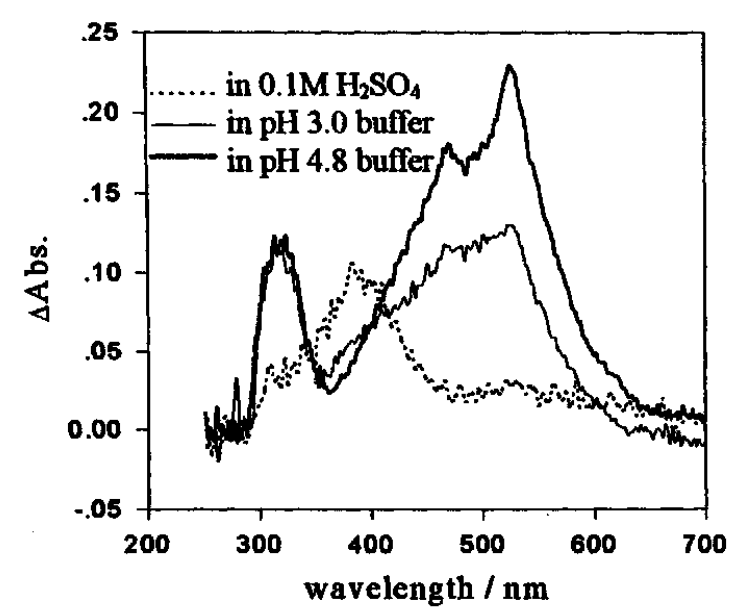

Fig. 6 Differences in absorption spectra between a spectrum obtained at open circuit and a spectrum obtained at applied potentials ( $700 \mathrm{mV}$ at $\mathrm{pH} 1.0,650$ $\mathrm{mV}$ at $\mathrm{pH} 3.0,550 \mathrm{mV}$ at $\mathrm{pH} 4.8$ ) in various $\mathrm{pH}$ solutions.

When no potential was applied only one band at 290 nm responsible for the dihydorxylbenzene moiety appeared in various $\mathrm{pH}$ solutions. There are three absorption bands $315,470,525 \mathrm{~nm}$, which are not observed at pH 1.0 solution when the potentials are applied. Also, absorbance of three bands is larger at pH 4.8 than at pH 3.0, indicating that the cyclized products through a chemical reaction form rapidly at higher $\mathrm{pH}$ solutions.

After bulk electrolysis in the same cell with a platinum gauze electrode, the recorded spectrum indicates that more than one products may be produced in the higher $\mathrm{pH}$ solution. Comparing all absorption peaks in $\mathrm{pH} 3.0$ and $\mathrm{pH} 4.8$ solution, there are at least two additional absorption bands which are strongly dependent on the $\mathrm{pH}$ of the solution. The peaks are of interest at 380 and $610 \mathrm{~nm}$. The absorption band at $610 \mathrm{~nm}$ was observed at pH 3.0 solution only and very weak band at $\mathrm{pH} 4.8$ and no band at $\mathrm{pH} 1.0$ solution.

The absorption spectra are not likely to be changed with time after electrolysis at $\mathrm{pH} 4.8$ at the open circuit. The strong absorption band at $610 \mathrm{~nm}$ (not shown) appeared when the potential was set to a negative potential than the $\mathrm{Cl}$ peak. The intensity of this band dramatically decreases by applying potentials greater than $50 \mathrm{mV}$. Therefore, this suggests that the $\mathbf{A 2}$ peak is due to the oxidation of a reduced form of ADQC, i.e., LAD. This is not in agreement with the previous papers. They assigned the peak as the oxidation of dehydrated form of LAD, i.e., IND; 5,6-dihydroxy-N-methylindole.
The difference spectra between the one obtained with an open circuit and the other obtained by applying an oxidation potential enough to oxidize the epinephrine in various pH solution are shown in Fig. 6. Formation of $\mathrm{ADQC}$ in higher $\mathrm{pH}$ solution was confirmed by two absorption bands at 315 and 470 $\mathrm{nm}$, which agrees with a reported value of 302 and $485 \mathrm{~nm}$ of ADQC. The smaller absorption band at $380 \mathrm{~nm}$ in $\mathrm{pH} 4.8$ solution than at more acidic solutions indicates that the $A D Q H$ converts quickly to the cyclized product of ADQC and/or LAD. Also, this assumption is also supported by absence of the C2 peak in Fig. 3b. Because the first oxidation product, $A D Q H$, returns quickly to epinephrine in acidic environment the formation of $\mathrm{ADQ}$ form is not likely to occur and the LAD is difficult to be formed from the $\mathrm{ADQH}$.

In conclusion, we demonstrate that our NNIRS system can be used to study electrochemical oxidation of epinephrine. It is not likely to produce the dehydrated form of LAD even in acidic condition. Further studies are under investigation to reveal the detailed mechanism on the oxidation of epinephrine.

\section{Acknowledgment}

This work was financially supported by Korea Science and Engineering Foundation (Contract No. 95-0501-05-01-3)

\section{References}

1. G. Dryhurst, "Comprehensive Treatise of Electrochemisty", Vol. 10, Chap. 2, Plenum Press, New York, 1985.

2. R. M. Wightman, L. J. May, and A. C. Michael, Anal. Chem., 60, 769A (1988).

3. A. Sucheta and F. J. Rusling, Electroanalysis, 3, 735(1991).

4. M. R. Deakin, P. M. Kovach, K. J. Stutts, and R. M. Wightman, Anal. Chem., 58, 1474 (1986).

5. X. Wu, L. Mu and W. Zhang, J. Electroanal. Chem., 352, 295(1993).

6. M. D. Hawley, S. V. Tatawawadi, S. Piekarski, and R. N. Adams, J. Am. Chem. Soc., 89, 447 (1967).

7. M. D. Hawley and S. W. Feldberg, J. Phys. Chem., 70, 3459 (1966).

8. C-H. Pyun and S-M. Park, Anal. Chem., 58, 251 (1986).

9. J. P. Skully and R. L. McCreery, Anal. Chem., 52, 1885 (1980). 